\title{
Molecular dynamics analysis of amorphous ice transformations: elastic properties and nucleation
}

Anastasiia Garkul ( $\square$ garkul.aa@phystech.edu )

Joint Institute for High Temperatures

Vladimir Stegailov

Joint Institute for High Temperatures

Research Article

Keywords:

Posted Date: February 2nd, 2022

DOI: https://doi.org/10.21203/rs.3.rs-1300246/v1

License: (c) (1) This work is licensed under a Creative Commons Attribution 4.0 International License. Read Full License 


\title{
Molecular dynamics analysis of amorphous ice transformations: elastic properties and nucleation
}

\author{
Anastasiia Garkul ${ }^{1,2, *}$ and Vladimir Stegailov ${ }^{1,2,3}$ \\ ${ }^{1}$ Joint Institute for High Temperatures of the Russian Academy of Sciences \\ ${ }^{2}$ Moscow Institute of Physics and Technology (National Research University) \\ ${ }^{3}$ National Research University Higher School of Economics \\ *garkul.aa@phystech.edu
}

\begin{abstract}
Unlike conventional first-order phase transitions, the kinetics of amorphous-amorphous transitions has been much less studied. The ultrasonic experiments on the transformations between low-density and high-density amorphous ice induced by pressure or heating provided the pressure and temperature dependencies of elastic moduli. In this article, we make an attempt to build a microscopic picture of these experimentally studied transformations using the molecular dynamics method with the TIP4P/lce water model. We study carefully the dependence of the results of elastic constants calculations on the shear and compression rates. The system size effects are considered as well. The comparison with the experimental data enriches our understanding of the transitions observed. Our modelling gives new information on the nucleation mechanisms during transformations between low-density and high-density amorphous ices and, thus, helps answering the question about first-order nature of this transition.
\end{abstract}

\section{Introduction}

Water is central to many physical, biological and industrial applications and exhibits physical properties that are qualitatively different from those of most other liquids. Despite the huge amount of theoretical and experimental research aimed at studying the many specific properties of water, it continues to be one of the most mysterious pure substances in the Universe. Supercooled water, known for its polymorphism, attracts a lot of interest. Today the phase diagram of water boasts no less than 19 modifications $^{1}$ of crystalline ice and 3 amorphous forms (not counting their derivatives) ${ }^{2}$, the most popular of which are the low density amorphous ice (LDA) and the high density amorphous ice (HDA). Amorphous ice practically does not occur on Earth in nature, but LDA is the most widespread form of $\mathrm{H}_{2} \mathrm{O}$ in the Universe, as it is a part of interstellar dust and comet nuclei. LDA can be obtained from the gas phase ${ }^{3}$ or from liquid water ${ }^{4}$.

Actually, the discovery by Mishima et al. ${ }^{5}$ of solid-state amorphization (SSA) Ih $\rightarrow$ HDA in 1984 launched the entire epoch of the studies of the nature of these non-equilibrium transformations. It is noteworthy that amorphous forms of water were obtained from stable phases of all three states of aggregation ${ }^{6}$. Under isothermal compression of LDA or of hexagonal ice Ih, a transition occurs, which leads to the formation of HDA, and LDA can then be restored from HDA under isothermal decompression.

An important question about the nature of amorphous ices is the hypothesis of the two-liquid model of water and the existence of a second critical point. One ${ }^{11}$ of several ${ }^{11-13}$ theoretical scenarios to explain water anomalies (such as a sharp increase in its isothermal compressibility, isobaric heat capacity, coefficient of the thermal expansion upon cooling below the equilibrium freezing point) postulates the existence of a first-order-like transition between two forms of liquid water that correspond to LDA and HDA below the glass transition line (Fig. 1) and ends at the liquid-liquid critical point (LLCP). It is assumed that LLCP is located in the area called the "no man's land" corresponding to the temperature range of $\sim 145-200 \mathrm{~K}$, where only crystalline forms of water are observed in experiments due to very rapid crystallization. With all the abundance of experimental observations ${ }^{14-19}$ that indirectly indicate the possibility of the existence of the liquid-liquid phase transition in supercooled water, not a single unambiguous experiment has proved this thesis yet. Experimental problems under these conditions are associated with the inevitable rapid crystallization of a metastable liquid state, although the recent study by Kim et al. ${ }^{20}$ provides a convincing evidence.

Molecular modelling plays an important role in studying the behavior of water under such conditions ${ }^{7,21-23}$. It allows heating at sufficiently high rates to avoid crystallization and at the same time to study the structure in detail. Moreover, some models of water demonstrate the liquid-liquid transition, others do not ${ }^{24,25}$. The TIP4P class of water models provides a well balanced instrument that allows predictive modelling of water-based systems at reasonable computational expenses and, therefore, at relatively large length and time scales (see e.g. ${ }^{26-29}$ ). 


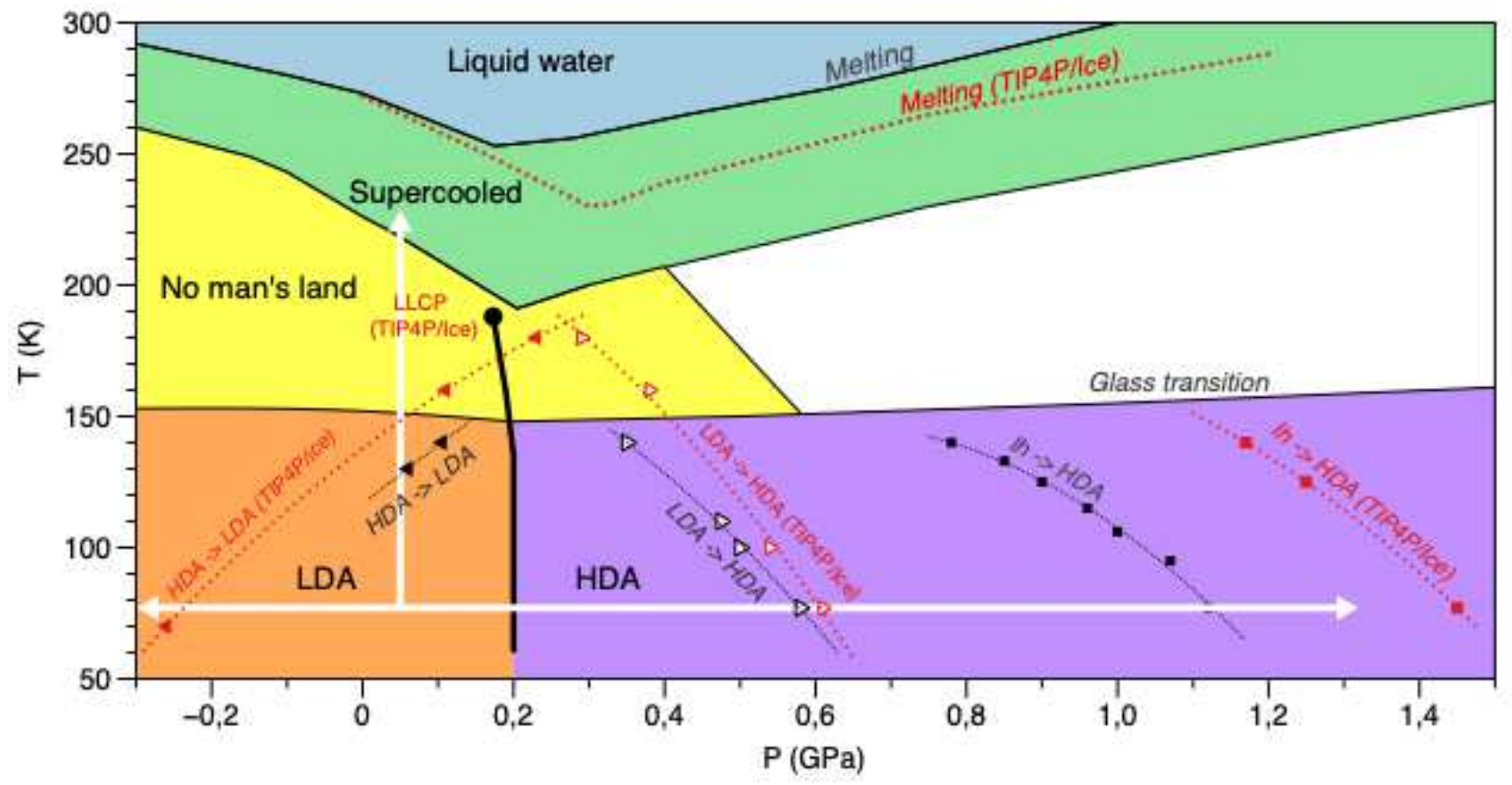

Figure 1. The phase diagram of amorphous and liquid water. The separation line between a low density liquid and a high density liquid ends at the liquid-liquid critical point (LLCP) ${ }^{7}$. This point is located in an area called "no Man's Land", marked in yellow, where only crystalline forms of ice are experimentally observed. Transition lines based on experimental work ${ }^{8,9}$ are indicated by black dotted lines; for the TIP4P/Ice model, the lines are in red dotted lines ${ }^{10}$. The white arrows show the paths considered in our work.

Various approaches are used to study structure of various amorphous ices: an analysis of partial radial distribution functions $(\mathrm{RDFs})^{30,31}$, the structural factor ${ }^{32}$, the classification using neural networks ${ }^{23}$. In this work, we rely on the partial RDF O-O and on the distribution of angles between hydrogen-bonded oxygen atoms that reflects the order in the tetrahedral structure. It was shown ${ }^{32}$ that the tetrahedral structure is distorted in the $\mathrm{Ih} \rightarrow \mathrm{LDA} \rightarrow \mathrm{HDA} \rightarrow$ liquid water sequence.

One of the tasks of this work is to form a microscopic understanding of the close relationship between elastic properties and the nature of a dynamic disorder present in amorphous ices. Our interest in studying the behavior of elastic properties in transformations between amorphous forms of ice is caused by the experimental works, where elastic moduli were measured by the ultrasonic method ${ }^{8}$. It was shown that elastic softening precedes amorphous-amorphous and crystal-amorphous transformations. But the theoretical interpretation of these results is still absent and has not been considered in any molecular modelling studies yet.

\section{Methods}

\section{Simulation details}

In this work, we consider classical molecular dynamics modelling of amorphous ice using the TIP4P/Ice water model ${ }^{10}$. Positions of particles are determined from the solution of the classical Newtonian equations of motion. The simulations are carried out for a system of $N=2880, N=23040$ and $N=77760$ water molecules, in a cubic box with periodic boundary conditions using the LAMMPS software package ${ }^{33}$ with the GPU acceleration for TIP4P models ${ }^{34,35}$. The cutoff radii for the Coulomb and Lennard-Jones interactions are $r_{c, \text { Coul }}=10 \AA$ and $r_{c, L J}=12 \AA$, respectively. The long-range electrostatic interactions are treated using the Particle-Particle-Particle-Mesh algorithm ${ }^{36}$ with an accuracy of $10^{-5}$. The SHAKE algorithm controls the bond lengths and angles for rigid molecules. The integration time step is $2 \mathrm{fs}$. The length of molecular dynamics trajectories is from $1 \mathrm{~ns}$ to $100 \mathrm{~ns}$. The calculations are performed in $N V T$ (the Nose-Hoover thermostat) and $N P T$ ensembles (the Nose-Hoover barostat). The visualization and structure analysis are carried out in the Ovito software package ${ }^{37}$.

\section{Elastic moduli}

Suzuki et. $\mathrm{al}^{38}$ note that it happens that for the same material, different values of elastic moduli are obtained, depending on the methods used to calculated them. In addition, it may turn out that the same method is in good agreement with experimental data 
for substances with a structure of one type, but for another structure it already poorly reproduces what is obtained in practice. This clearly demonstrates the limits of applicability of one method or another. Meanwhile, it is important how well the model used describes the properties of a real system, in comparison with alternative ones, especially for water, for which more than a hundred different models have been proposed, but each is used for specific purposes ${ }^{26}$. Unlike other works on molecular dynamics modelling $^{38,39}$, which study elastic properties with special emphasis on structural phase transitions, where elastic moduli are calculated using the fluctuation formula of molecular dynamics, here we calculate the elastic moduli directly ${ }^{40}$.

The definition of the isothermal bulk modulus of elasticity is given by:

$$
B=-V\left(\frac{\partial P}{\partial V}\right)_{T}=\rho\left(\frac{\partial P}{\partial \rho}\right)_{T},
$$

where $P$ is the pressure, $V$ is the volume, $\rho$ is the density of the system. To find the bulk modulus, after equilibration in the $N V T$ ensemble for at least $2 \mathrm{~ns}$, it is necessary to compress the simulation box by changing the linear dimensions by $\pm 1 \%$ (while the deformation is linear) in each of the three directions and find the slope coefficient $P(\rho)$.

The shear modulus is by definition:

$$
G=-\frac{d \tau_{x y}}{d \gamma_{x y}}
$$

where $\tau_{x y}$ is the shear stress, $\gamma_{x y}$ is the shear strain.

To calculate the shear modulus $G$, by analogy with the bulk modulus $B$, we carry out a shear strain of $\pm 1-2 \%$ in one direction. $G$ is found as the slope coefficient $\tau(\gamma)$. It is important that our system is isotropic with respect to the shear modulus. We have carried out three independent deformations in different directions and made sure that the result does not depend on the direction of the shear (see Supplementary Fig. S1 online). The values of the elastic moduli for each state $(P, T)$ are obtained by averaging over 3-5 independent straining simulations.

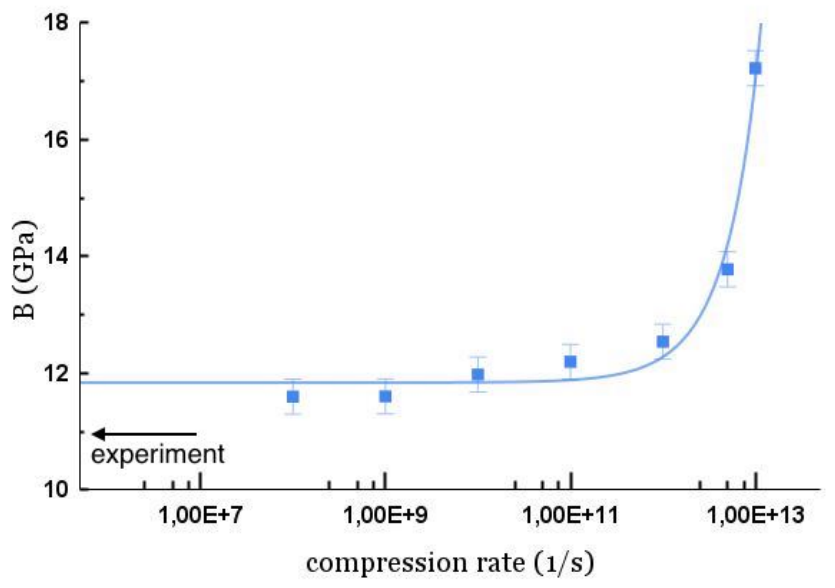

a)

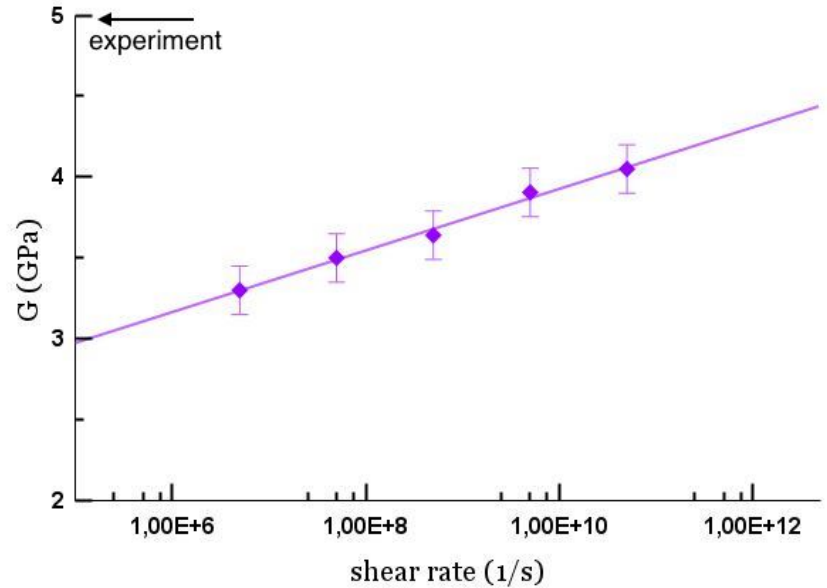

b)

Figure 2. The dependence of elastic moduli of HDA on the relative strain rate. a) The compression rate dependence of the bulk modulus at $T=77 \mathrm{~K}$ and $P=0.17 \mathrm{GPa}$; the solid blue line shows an exponential interpolation. b) The shear rate dependence of the shear modulus at $T=77 \mathrm{~K}$ and $P=0.05 \mathrm{GPa}$; the solid purple line shows a logarithmic interpolation. The abscissa axis is in the logarithmic scale. The experimental values ${ }^{8}$ are shown by arrows.

In addition, the strain rate can be varied, but this affects the values of the elastic moduli. Fig. 2 shows the values of $B$ and $G$ for calculations with different rates of relative deformation under the same conditions. For the bulk modulus (Fig. 2 (a)), exponential convergence is observed with decreasing compression rate. Moreover, for lower velocities, the value is closer to the experimental one ${ }^{8} B_{\text {exp }}=11 \mathrm{GPa}$, where the elastic moduli are found by the ultrasonic method at frequencies of $5 \mathrm{MHz}$. Actually, the ultrasonic method involves the measurement of adiabatic characteristics, so that the slight difference in values is probably due to the fact that we consider the isothermal modulus. Thus, there is not much benefit in using very low compression rates (less than $10^{9} \mathrm{~s}^{-1}$ ). The behavior of the strain rate dependence of the shear modulus is different. No convergence is observed in this case, and there is a significant discrepancy (about 30\%) with the experimental value $G_{\text {exp }}=5$ GPa. Further, shear modulus calculations are carried out at a shear deformation rate of $5 \cdot 10^{7} \mathrm{~s}^{-1}$. 
We are able to calculate the bulk modulus of amorphous ice with a deviation of less than $10 \%$ from the experimental value, while for hexagonal ice we have a deviation of about $70 \%$, this is consistent with the work Moreira et al. ${ }^{41}$. They took a similar approach using several water models, TIP4P/Ice including.

\section{Preparatory stage}

\section{Preparation of LDA}

We obtain LDA by fast isobaric cooling of liquid water at a rate of $10 \mathrm{~K} / \mathrm{ns}$. In general, water is a poor glass former, and in practice a cooling rate of more than $0.01 \mathrm{~K} / \mathrm{ns}$ is required to avoid crystallization. It was also shown ${ }^{42}$ that the structure of the obtained amorphous ice changes weakly with a variation in the cooling rate in the range of $0.1-1 \mathrm{~K} / \mathrm{ns}$, the density deviations are within statistical error. As a result, we get a system at $T=77 \mathrm{~K}$ and $P=0.01 \mathrm{MPa}$ with a density of $0.95 \mathrm{~g} / \mathrm{cm}^{3}$, whereas in the experiment under such conditions the density is $0.94 \mathrm{~g} / \mathrm{cm}^{3}$. As can be seen in Fig. 3, the radial distribution function is in good qualitative agreement with the experimental one ${ }^{30}$, but the first peak is overestimated, which was already shown earlier ${ }^{10}$ for the TIP4P/Ice liquid state.

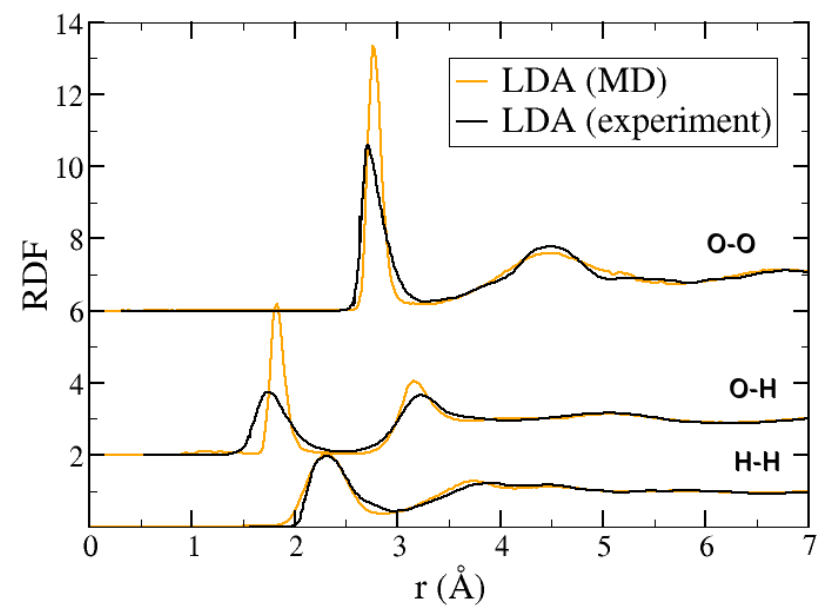

a)

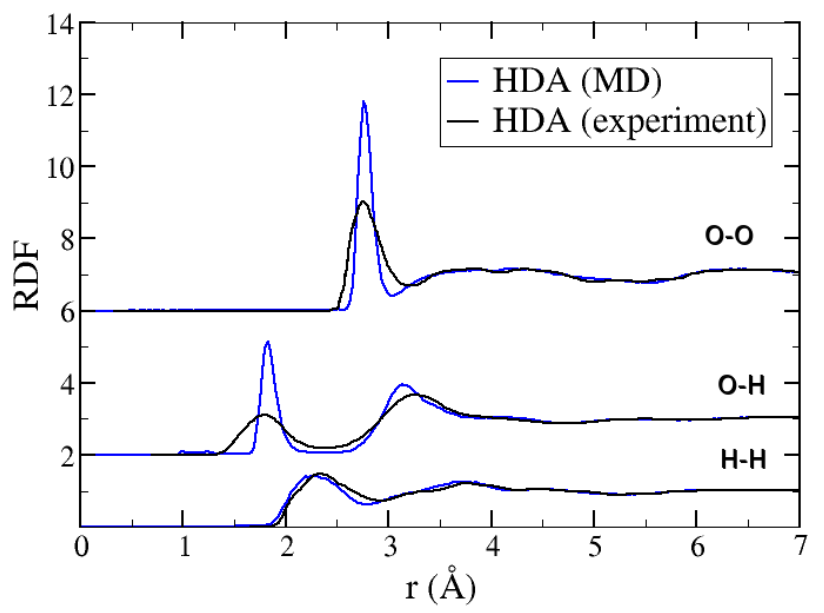

b)

Figure 3. The radial distribution functions of LDA (a) and HDA (b) at $T=77 \mathrm{~K}$ and $P=0.1 \mathrm{MPa}$ for TIP4P/Ice water model and the experimental data ${ }^{30}$.

\section{Preparation of HDA}

To obtain HDA, hexagonal ice at a temperature of $77 \mathrm{~K}$ and a pressure of $0.1 \mathrm{MPa}$ is isothermally compressed (NVT ensemble, compression is performed by decreasing the size of the computational cell linearly with time) from a density of $0.95 \mathrm{~g} / \mathrm{cm}^{3}$ to $1.31 \mathrm{~g} / \mathrm{cm}^{3}$. After equilibration the system pressure is $1.4 \mathrm{GPa}$. Upon further release of the pressure $0.01 \mathrm{MPa}$, the amorphous structure is retained, the system remains metastable. An amorphous form is actually obtained that differs from LDA, its density at $T=77 \mathrm{~K}$ and $P=0.01 \mathrm{MPa}$ is $1.15 \mathrm{~g} / \mathrm{cm}^{3}$. Moreover, RDF of HDA has noticeable differences (Fig. 3). The second HDA's peak has a broader shape and a slight splitting at $4 \AA$. Also, there is an increased probability of finding water molecules at an O-O distance of 3.0-3.5 $\AA$ from the central water molecule. We repeated the calculation for a larger system, but no significant size effect is observed (see Supplementary Fig. S2 online).

In the experiment, SSA of hexagonal ice occurs already at $1.1 \mathrm{GPa}$, and here the compression rate plays a certain role. We consider isothermal compression with different compression rates in the range of 0.1-5 GPa/ns. In Supplementary Fig. S2 online dependencies of $P(\rho)$ for different compression rates are given. The amorphization pressure decreases with decreasing compression rate. At the same time, our lowest compression rate $q=0.1 \mathrm{GPa} / \mathrm{ns}$ (in terms of density, this corresponds to a compression rate of $0.05 \mathrm{~g} / \mathrm{cm}^{3} / \mathrm{ns}$ ), which is several orders of magnitude higher than the experimental rate ${ }^{8} 10^{-12} \mathrm{GPa} / \mathrm{ns}^{-}$It is problematic to mimic the compression rate in molecular dynamics as in the experiment, nevertheless, the amorphization pressure obtained by us in the MD model is extrapolated to the corresponding experimental value well . 


\section{Results}

\section{Pressure-induced transformations}

In this section of our study, we carry out isothermal $(T=77 \mathrm{~K})$ compression/decompression of amorphous ices. For the initial system, we take the LDA obtained earlier (point 1 in Fig. 4 (a)) and compress it at a rate of $0.1 \mathrm{GPa} / \mathrm{ns}$ to a pressure of $2 \mathrm{GPa}$ (point 3). Then the pressure is gradually released, until negative values (point 6), and compression begins again. Thus, in our model, transformations between amorphous forms of ice occur in a cycle that resembles "hysteresis", as in experiments 8,9 .

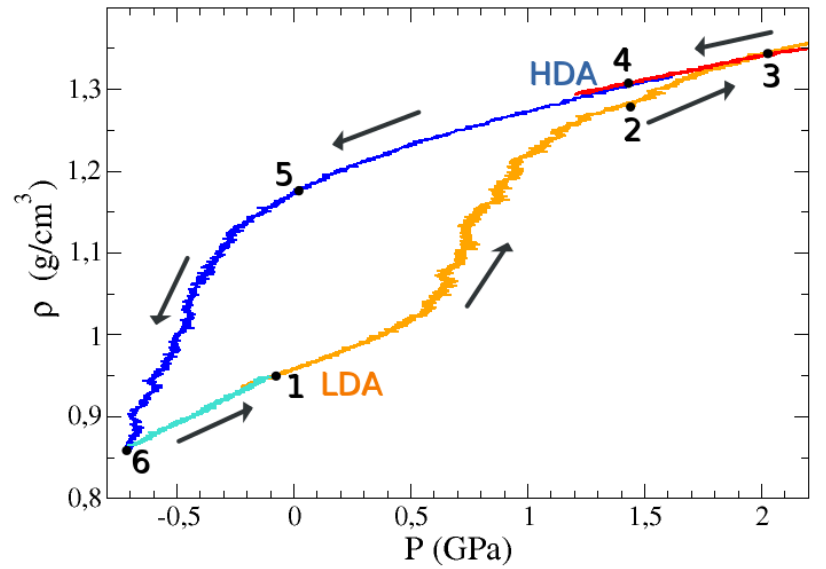

a)

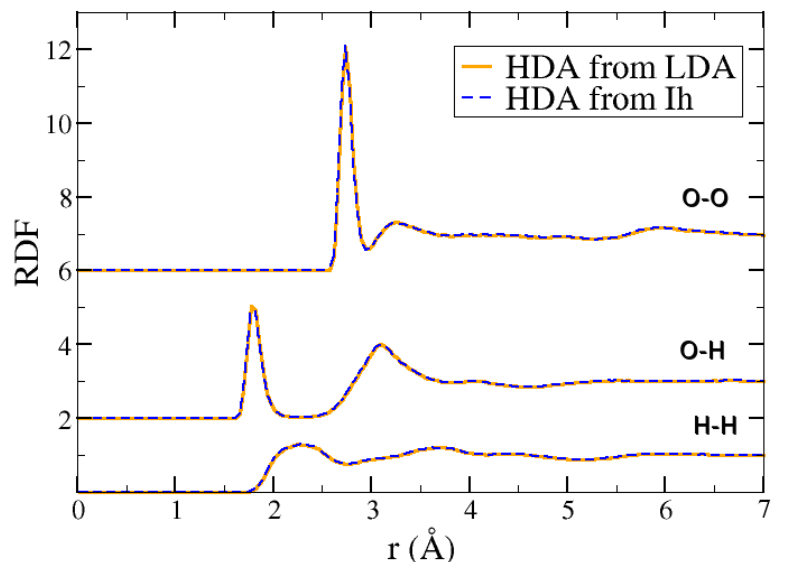

b)

Figure 4. a) The pressure dependence of density during the transformations of amorphous ices under pressure at $T=77 \mathrm{~K}$, the compression rate is $0.1 \mathrm{GPa} / \mathrm{ns}$. The arrows indicate the cycle direction. On the route $4 \rightarrow 5$, the blue solid line is the decompression of HDA obtained during SSA from Ih, the red line is the decompression of HDA obtained from LDA. b) The comparison of the partial RDFs of HDA obtained by SSA from Ih and by compression from LDA.

In fact, in MD we have implemented two methods for obtaining HDA: from hexagonal ice and from LDA (point 4 in Fig. 4 (a)). Comparing the radial distribution functions of the resulting structures (Fig. 4 (b)), we can conclude that the HDA structure is independent of the method of its formation for these two cases considered.

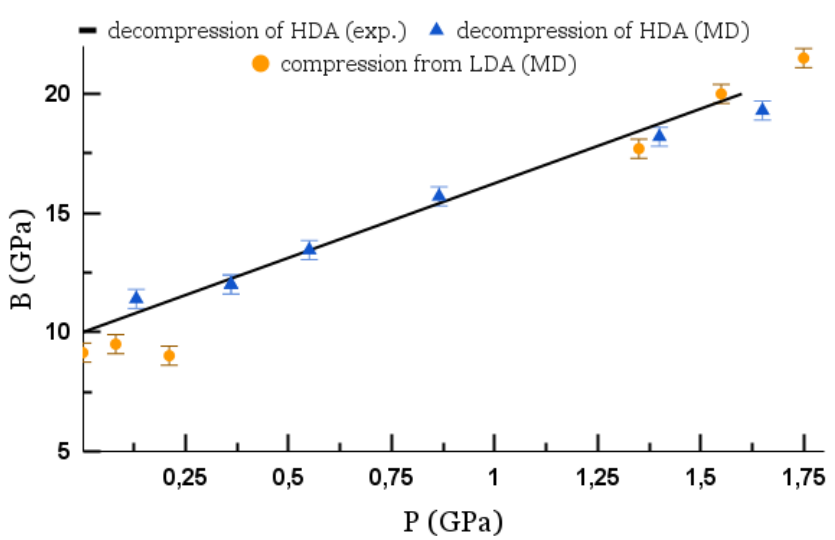

a)

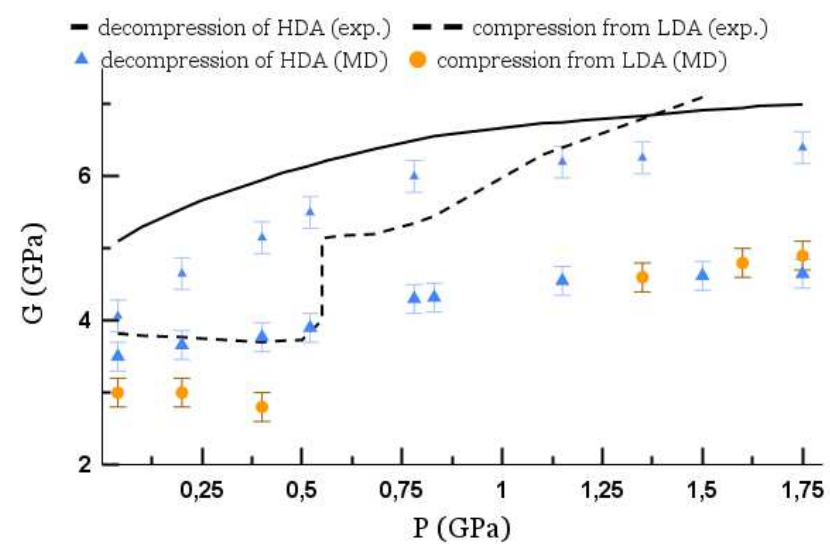

b)

Figure 5. The pressure dependence of elastic moduli during isothermal $(T=77 \mathrm{~K})$ compression and decompression at a compression rate $0.1 \mathrm{GPa} / \mathrm{ns}$ : a) the bulk modulus, b) the shear modulus. The dotted line corresponds to the experimental data during LDA compression at $110 \mathrm{~K}$. The small blue symbols correspond to the results of the calculation with a higher shear rate of $5 \cdot 10^{10} \mathrm{~s}^{-1}$ during decompression of HDA.

By analogy with a phase transition, the transition from LDA to HDA implies a change in local structure and should lead to a change of elastic characteristics. The dependence of the bulk modulus of elasticity on pressure during HDA decompression is shown in Fig. 5. We also calculate the bulk modulus of LDA at the beginning and at the end of the compression, but this method does not allow finding intermediate values, since at 0.5-1.5 GPa the system is unstable. Nevertheless, one can be convinced that 


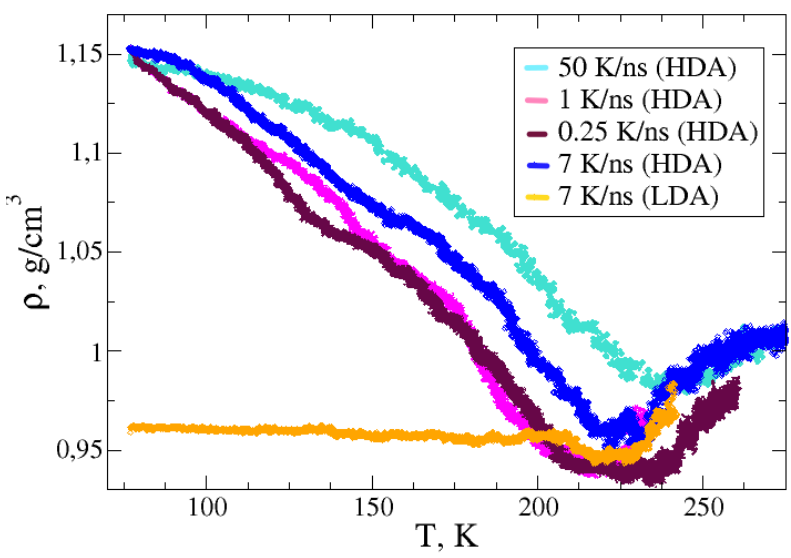

a)

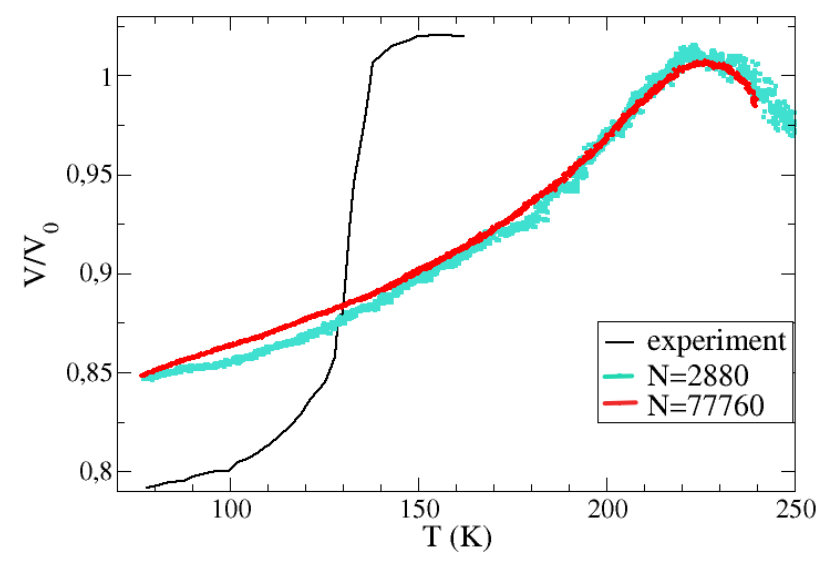

b)

Figure 6. a) The temperature dependencies of density during the isobaric heating of HDA for several heating rates. The orange line shows the dependence in the process of heating of LDA at the rate of $7 \mathrm{~K} / \mathrm{ns}$. b) The absence of the size effect during isobaric $(P=0.05 \mathrm{GPa})$ heating of HDA. The MD-heating rate is $7 \mathrm{~K} / \mathrm{ns}$; the experiment ${ }^{8}$ assumes much slower heating.

upon compression of LDA, the elastic modulus increases to values similar to HDA. During HDA decompression, the result of our model is in good agreement with the experiment ${ }^{8}$ for the pressure dependence of bulk modulus. For the shear modulus, we have a slightly larger quantitative difference.

\section{Heating-induced transformations}

Before we start discussing isobaric heating of amorphous ices, it is necessary to understand what rate of heating makes it possible to obtain an adequate description at a not very high computational costs. To do this, we carry out several processes of HDA heating at constant pressure $(0.05 \mathrm{GPa})$ at different rates in the range $Q_{\text {heat }}=0.25-50 \mathrm{~K} / \mathrm{ns}$, and Fig. 6 (a) shows how the density behaves with increasing temperature. At a heating rate of less than $7 \mathrm{~K} / \mathrm{ns}$, the curves almost converge, and for further analysis we will consider heating at a rate $Q_{\text {heat }}=7 \mathrm{~K} / \mathrm{ns}$.

Thus, the temperature dependencies of density of LDA and HDA under isobaric heating at the same rate is shown in Fig. 6 (a). And as it is not difficult to see, at $T>210 \mathrm{~K}$ the densities of the systems become almost the same. Moreover, at a temperature of $240 \mathrm{~K}$, the structures of heated LDA and HDA are identical, which is indicated by the similarity of the RDF and the distribution of angles between oxygen atoms (see Supplementary Fig. S3 online)), that is, we can say that the transformation of LDA and HDA into liquid water has occurred.

A more interesting question is what happens to amorphous ices before the transition to the liquid water phase? Trying answering this question, we compare the structure of the systems at $77 \mathrm{~K}$ and $180 \mathrm{~K}$. How the RDF and the tetrahedral structure change can be seen in more detail in Supplementary Fig. S3 online. Up to $180 \mathrm{~K}$, an increase in the peak of the angular distribution is observed, from which it can be concluded that, before the liquid phase, the HDA passes into an LDA-like state. Since the RDF is an ensemble-averaged characteristic of the system, the intermediate states between 90 and $180 \mathrm{~K}$ are more likely to relate to a mixture of two phases, where HDA first prevails, and then LDA.

It should be mentioned that under the experimental conditions at HDA heating, a rather sharp jump of $15 \%$ in the density (or specific volume) occurs at a temperature of $130-140 \mathrm{~K}^{8}$, which was attributed by authors precisely to the HDA-LDA transition. In our modelling, however, the specific volume increases gradually (Fig. 6 (b)). This fact is can be associated with both the high heating rate and the use of the barostat, which facilitates the processes of local structural rearrangements. Besides, the behavior of the temperature dependencies of specific volume for systems with 2880 and 77760 molecules is practically the same.

As for the temperature dependencies of the elastic moduli (Fig. 7), we obtain a fairly good quantitative agreement with experiment ${ }^{8}$ for the bulk modulus; however, we do not observe qualitatively no-monotony behavior in the model, as well as crystallization into cubic ice at $160 \mathrm{~K}$ with which these peculiarities are associated. The calculation of the shear modulus does not give such a good quantitative agreement. Nevertheless, both characteristics reflect softening of the amorphous network of LDA and HDA. The relatively sharp decrease in the bulk modulus of LDA at $190 \mathrm{~K}$ is most likely precedes further transformation into liquid water. And the decrease in the shear modulus at temperatures above $225 \mathrm{~K}$ to practically zero values is consistent with the fact that both systems transform into the liquid phase. 


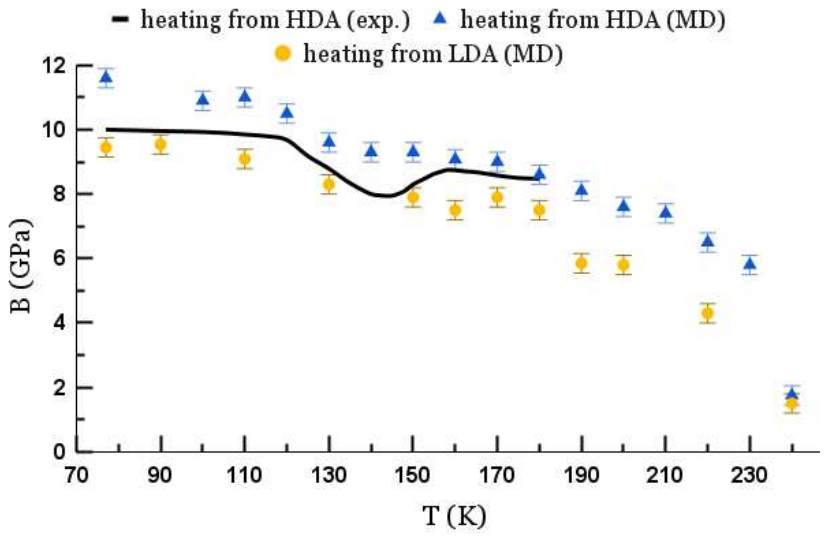

a)

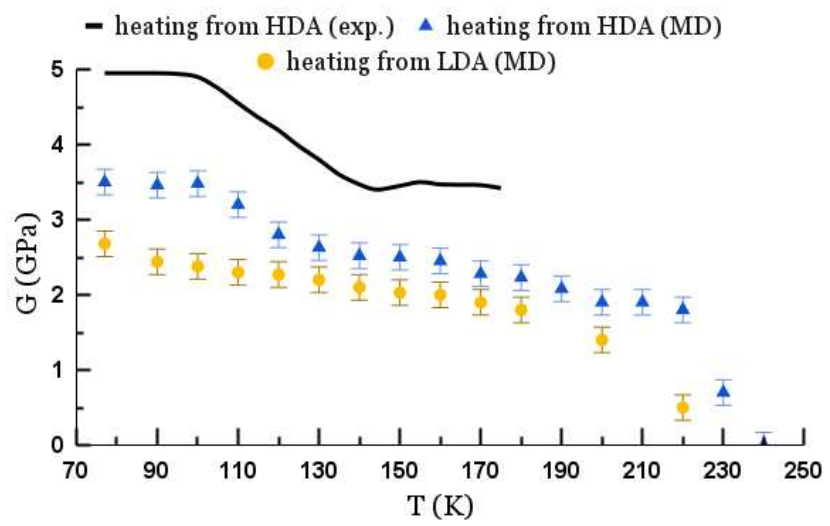

b)

Figure 7. The temperature dependencies of a) the bulk modulus and b) the shear modulus during isobaric heating $(P=0.05 \mathrm{GPa})$.

\section{Nucleation during LDA $\leftrightarrow$ HDA transitions}

We made an attempt to see nucleation, using the fact that in HDA phase for an oxygen atom, the probability of detecting a neighboring oxygen atom at a distance of 3.0-3.5 $\AA$ increases. We classify molecules with a reduced probability of finding a neighbor at a distance of 3.0-3.5 $\AA$ as an LDA-like state and molecules with an increased probability as HDA. First, in the process of the LDA $\rightarrow$ HDA transition under isothermal compression $(T=77 \mathrm{~K})$, the number of molecules characteristic of HDA increases with increasing pressure; in addition, growing HDA clusters are formed, which is shown in Fig. 8 (a). The size of the critical nucleus can be estimated as 5 molecules. That is, the nucleation takes place.

Next, let us consider the inverse transformation HDA $\rightarrow$ LDA during isothermal decompression to negative pressures. By analogy with the previous case, the formation of growing LDA clusters is observed as well (Fig. 8 (b)).

But during isobaric heating $(P=0.05 \mathrm{GPa})$ of HDA, tiny LDA clusters of 2-3 molecules are distributed homogeneously in the simulation box and their number gradually increases without the formation and growth of critical nuclei (see Supplementary Fig. S4 online)). Therefore, in this case there is no sign of nucleation during HDA $\rightarrow$ LDA transition. In order to consider possible system size effects, we perform the same modelling for the system of 77760 molecules, but the nature of the transition remains qualitatively the same without any visible nucleation and growth events. The absence of nucleation during this isobaric heating can be explained by the nearly constant level of metastability along the isobar that goes parallel to the LDA-HDA equilibrium line.

\section{Discussion}

In this work using the MD modelling with the TIP4P/ice potential we have confirmed that the model gives a consistent description of LDA and HDA amorphous ices and have reproduced the key experimental results on LDA $\leftrightarrow$ HDA transitions ${ }^{8}$ :

1. There is a satisfactory agreement with experimental results ${ }^{30}$ for the RDFs of LDA and HDA. The extra height of the first peaks of RDFs in the TIP4P/Ice molecular dynamics tells that the O-O and H-O bonds are too rigid. This fact, however, is a well-known feature of classical water models that do not take into account nuclear quantum effects at low temperatures ${ }^{43,44}$.

2. During the isothermal SSA of Ih into HDA, the amorphization pressure depends on the compression rate, but no significant size effect was observed. The MD results are varying (very high) compression rates show a reasonable convergence to the experimental results.

3. The non-equilibrium modelling of pressure-induced transformations between LDA and HDA reflects the main features observed experimentally: the jump in density at the LDA $\rightarrow$ HDA transition and the hysteresis. The reverse transition of the HDA $\rightarrow$ LDA during decompression is observed at negative pressures. The RDFs of HDA structures obtained from Ih and from LDA are indistinguishable.

4. We have analyzed the compression rate and share rate dependencies in the MD calculations of elastic moduli. We show that the bulk modulus converges quickly at decreasing compression rate. However, no convergence has been detected for the shear modulus at decreasing shear rate. Therefore, the calculations have been performed with the shear rate close to the corresponding ultrasonic frequency in the experiment ${ }^{8}$. The results of the corresponding MD calculations of bulk and shear moduli of HDA are in a good overall agreement with the experimental data ${ }^{8}$ for the case of isothermal compression and for the case of isobaric heating. There is a very good quantitative agreement for the bulk modulus (the accurate prediction of the liquid 


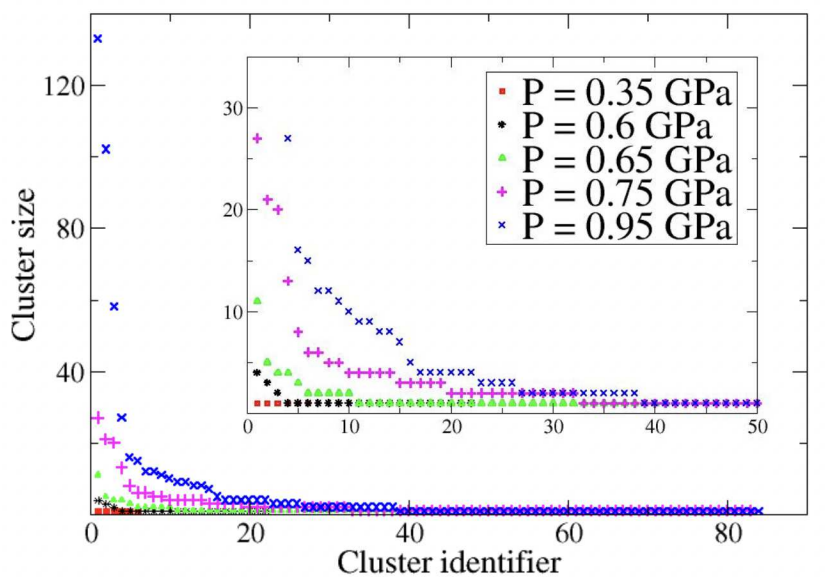

a)
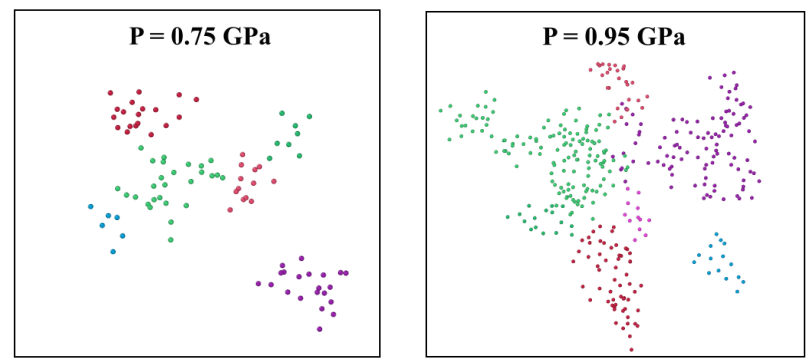

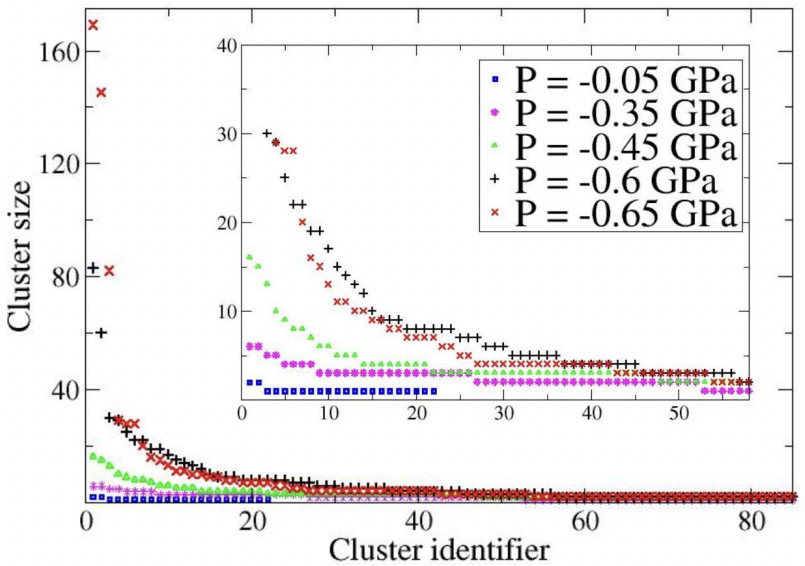

b)
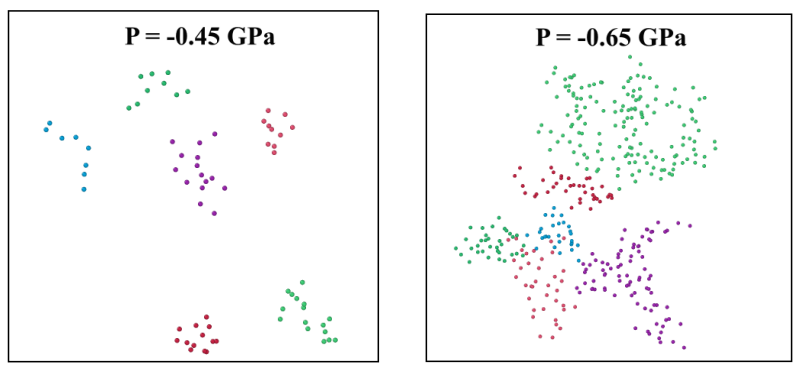

Figure 8. a) The formation of growing HDA clusters during isothermal compression LDA $\rightarrow$ HDA. b) The formation of growing LDA clusters during isothermal decompression HDA $\rightarrow$ LDA. The snapshots below show the largest clusters in the system at two time moments for each processes. In both cases, the total number of molecules in the system is 2880 and $T=77 \mathrm{~K}$.

water bulk modulus at room temperatures in TIP4P/2005 model has been reported recently as well ${ }^{45,46}$ ). However, the MD results for the shear modulus are about $50 \%$ lower that the experimental data. Here it is worth mentioning that the agreement for the TIP4P/Ice result for the bulk modulus of Ih is about $70 \%$ higher that the experimental value (similarly as it was shown previously ${ }^{41}$ ). It has been shown ${ }^{47,48}$ that including nuclear quantum effects in MD modelling improves the prediction of the Ih bulk modulus.

In the framework of the presented model, we have developed a nearest neighbours analysis method that distinguishes clusters of HDA in LDA and vice versa. Using this method, we have shown that both the LDA $\rightarrow$ HDA and the HDA $\rightarrow$ LDA transformations at isothermal compression/decompression proceed via the nucleation of a new phase as soon as a high degree of metastability is achieved. There are clear pictures of nucleation and growth events in the system of 2880 molecules for both transitions. This fact is an important argument in favor of the first order nature of the LDA $\leftrightarrow$ HDA transition.

The most problematic case for the MD modelling considered is the isobaric heating of HDA. In this, case we observe in MD modelling a gradual transition of HDA to LDA and then to liquid water. Contrary to the experimental data, in MD modelling there is no rapid increase of specific volume. The absence of nucleation events can be explained by the nearly constant level of metastability during this isobaric heating process: the system moves on the T-P diagram in parallel to the LDA-HDA equilibruim line. Moreover, the system approaches the region near the second critical point ${ }^{7}$. It is known that the size of critical nucleus becomes larger when the system approaches its critical point. Therefore, we can make a hypothesis that the proper explanation of the experimental data on the isobaric heating requires a theory for HDA-LDA-liquid water transformations at larger time and length scales well beyond the MD scale.

\section{Conclusions}

The TIP4P/Ice molecular dynamics modelling of amorphous ices and LDA $\leftrightarrow$ HDA transformations gives consistent results that have been compared with the ultrasonic measurements of elastic constants in HDA, LDA and during the LDA $\rightarrow$ HDA 
isothermal transformation and during the HDA $\rightarrow$ LDA isobaric transformation by Gromnitskaya et al. ${ }^{8}$ MD modelling gives the correct qualitative description of the pressure and temperature dependencies of bulk and shear moduli of HDA and LDA in these processes. The TIP4P/Ice water model provides a quantitatively accurate description for the HDA bulk modulus, but for the HDA shear modulus the absolute values are significantly lower that the experimental values.

Molecular dynamics modelling captures the essential features of the LDA $\leftrightarrow$ HDA transition observed experimentally. Therefore, the model is able to supplement the experiment and allows studying in details the reverse process of HDA $\rightarrow$ LDA isothermal transformation that takes place at negative pressures. Using this model, we have shown that both types of isothermal LDA $\leftrightarrow$ HDA transformations proceed via the nucleation and growth mechanism at high levels of metastability that confirms the first order nature of the LDA $\leftrightarrow$ HDA transition.

\section{Data availability}

The datasets obtained and analysed during the current study available from the corresponding author on reasonable request.

\section{References}

1. Loerting, T., Fuentes-Landete, V., Tonauer, C. M. \& Gasser, T. M. Open questions on the structures of crystalline water ices. Commun. Chem. 3, 1-4 (2020).

2. Loerting, T. et al. How many amorphous ices are there? Phys. Chem. Chem. Phys. 13, 8783-8794 (2011).

3. Burton, E. \& Oliver, W. The crystal structure of ice at low temperatures. Proc. Royal Soc. London. Ser. A-Mathematical Phys. Sci. 153, 166-172 (1935).

4. Brüggeller, P. \& Mayer, E. Complete vitrification in pure liquid water and dilute aqueous solutions. Nature 288, 569-571 (1980).

5. Mishima, O., Calvert, L. \& Whalley, E. Melting ice Ih at $77 \mathrm{~K}$ and $10 \mathrm{Kbar}$ : A new method of making amorphous solids. Nature 310, 393-395 (1984).

6. Widmann, G. \& Riesen, R. The glass transition of water and aqueous systems. J. thermal analysis calorimetry 52, 109-113 (1998).

7. Debenedetti, P. G., Sciortino, F. \& Zerze, G. H. Second critical point in two realistic models of water. Science 369, 289-292 (2020).

8. Gromnitskaya, E., Stal'gorova, O., Brazhkin, V. \& Lyapin, A. Ultrasonic study of the nonequilibrium pressure-temperature diagram of $\mathrm{H}_{2} \mathrm{O}$ ice. Phys. Rev. B 64, 094205 (2001).

9. Mishima, O., Calvert, L. \& Whalley, E. An apparently first-order transition between two amorphous phases of ice induced by pressure. Nature 314, 76-78 (1985).

10. Abascal, J., Sanz, E., García Fernández, R. \& Vega, C. A potential model for the study of ices and amorphous water: TIP4P/ice. The J. chemical physics 122, 234511 (2005).

11. Poole, P. H., Sciortino, F., Grande, T., Stanley, H. E. \& Angell, C. A. Effect of hydrogen bonds on the thermodynamic behavior of liquid water. Phys. review letters 73, 1632 (1994).

12. Speedy, R. J. Stability-limit conjecture. an interpretation of the properties of water. The J. Phys. Chem. 86, 982-991 (1982).

13. Sastry, S., Debenedetti, P. G., Sciortino, F. \& Stanley, H. E. Singularity-free interpretation of the thermodynamics of supercooled water. Phys. Rev. E 53, 6144 (1996).

14. Mishima, O. \& Stanley, H. E. Decompression-induced melting of ice IV and the liquid-liquid transition in water. Nature 392, 164-168 (1998).

15. Zanotti, J.-M., Bellissent-Funel, M.-C. \& Chen, S.-H. Experimental evidence of a liquid-liquid transition in interfacial water. EPL (Europhysics Lett. 71, 91 (2005).

16. Winkel, K., Elsaesser, M. S., Mayer, E. \& Loerting, T. Water polyamorphism: Reversibility and (dis)continuity. The J. chemical physics 128, 044510 (2008).

17. Clark, G. N., Hura, G. L., Teixeira, J., Soper, A. K. \& Head-Gordon, T. Small-angle scattering and the structure of ambient liquid water. Proc. Natl. Acad. Sci. 107, 14003-14007 (2010).

18. Amann-Winkel, K. et al. Water's second glass transition. Proc. Natl. Acad. Sci. 110, 17720-17725 (2013). 
19. Gallo, P. et al. Water: A tale of two liquids. Chem. reviews 116, 7463-7500 (2016).

20. Kim, K. H. et al. Experimental observation of the liquid-liquid transition in bulk supercooled water under pressure. Science 370, 978-982 (2020).

21. Moore, E. B. \& Molinero, V. Structural transformation in supercooled water controls the crystallization rate of ice. Nature 479, 506-508 (2011).

22. Palmer, J. C., Car, R. \& Debenedetti, P. G. The liquid-liquid transition in supercooled ST2 water: a comparison between umbrella sampling and well-tempered metadynamics. Faraday discussions 167, 77-94 (2013).

23. Martelli, F., Leoni, F., Sciortino, F. \& Russo, J. Connection between liquid and non-crystalline solid phases in water. The J. Chem. Phys. 153, 104503 (2020).

24. Limmer, D. T. \& Chandler, D. The putative liquid-liquid transition is a liquid-solid transition in atomistic models of water. The J. chemical physics 135, 134503 (2011).

25. Limmer, D. T. \& Chandler, D. The putative liquid-liquid transition is a liquid-solid transition in atomistic models of water.II. The J. chemical physics 138, 214504 (2013).

26. Vega, C. \& Abascal, J. L. Simulating water with rigid non-polarizable models: a general perspective. Phys. Chem. Chem. Phys. 13, 19663-19688 (2011).

27. Smirnov, G. S. \& Stegailov, V. V. Toward determination of the new hydrogen hydrate clathrate structures. The J. Phys. Chem. Lett. 4, 3560-3564 (2013).

28. Orekhov, M. Improving molecular dynamics calculation of diffusivity in liquids with theoretical models. J. Mol. Liq. 322, 114554 (2021).

29. Bakulin, I., Kondratyuk, N., Lankin, A. \& Norman, G. Properties of aqueous 1, 4-dioxane solution via molecular dynamics. The J. Chem. Phys. 155, 154501 (2021).

30. Finney, J., Hallbrucker, A., Kohl, I., Soper, A. \& Bowron, D. Structures of high and low density amorphous ice by neutron diffraction. Phys. review letters 88, 225503 (2002).

31. Bowron, D. et al. The local and intermediate range structures of the five amorphous ices at $80 \mathrm{~K}$ and ambient pressure: A Faber-Ziman and Bhatia-Thornton analysis. The J. chemical physics 125, 194502 (2006).

32. Subbotin, O., Belosludov, V., Inerbaev, T., Belosludov, R. V. \& Kawazoe, Y. Modelling of the structure and vibrational properties of LDA, HDA, and VHDA amorphous ices. Comput. materials science 36, 253-257 (2006).

33. Thompson, A. P. et al. LAMMPS - a flexible simulation tool for particle-based materials modeling at the atomic, meso, and continuum scales. Comput. Phys. Commun. 271, 108171 (2022).

34. Nikolskiy, V. \& Stegailov, V. GPU acceleration of four-site water models in LAMMPS. In Parallel Computing: Technology Trends, 565-573 (IOS Press, 2020).

35. Kondratyuk, N., Nikolskiy, V., Pavlov, D. \& Stegailov, V. GPU-accelerated molecular dynamics: State-of-art software performance and porting from nvidia CUDA to AMD HIP. The Int. J. High Perform. Comput. Appl. 35, 312-324 (2021).

36. Hockney, R. W. \& Eastwood, J. W. Computer simulation using particles (crc Press, 2021).

37. Stukowski, A. Visualization and analysis of atomistic simulation data with ovito-the open visualization tool. Model. Simul. Mater. Sci. Eng. 18, 015012 (2009).

38. Suzuki, T., Shimono, M., Ren, X. \& Wuttig, M. Molecular dynamics study of isothermal and adiabatic elastic moduli prior to martensitic transformation. J. alloys compounds 355, 183-187 (2003).

39. Kimizuka, H. \& Kaburaki, H. Molecular dynamics study of the high-temperature elasticity of $\mathrm{SiO}_{2}$ polymorphs: Structural phase transition and elastic anomaly. physica status solidi (b) 242, 607-620 (2005).

40. Mehl, M. J. Pressure dependence of the elastic moduli in aluminum-rich Al-Li compounds. Phys. Rev. B 47, 2493 (1993).

41. Franco Pinheiro Moreira, P. A., Gomes de Aguiar Veiga, R. \& de Koning, M. Elastic constants of ice ih as described by semi-empirical water models. The J. chemical physics 150, 044503 (2019).

42. Wong, J., Jahn, D. A. \& Giovambattista, N. Pressure-induced transformations in glassy water: A computer simulation study using the TIP4P/2005 model. The J. chemical physics 143, 074501 (2015).

43. Herrero, C. P. \& Ramírez, R. High-density amorphous ice: A path-integral simulation. The J. chemical physics 137, 104505 (2012). 
44. Kondratyuk, N., Norman, G. \& Stegailov, V. Quantum nuclear effects in water using centroid molecular dynamics. In Journal of Physics: Conference Series, vol. 946, 012109 (IOP Publishing, 2018).

45. Abascal, J. L. \& Vega, C. Widom line and the liquid-liquid critical point for the tip4p/2005 water model. The J. Chem. Phys. 133, 234502 (2010).

46. Eltareb, A., Lopez, G. E. \& Giovambattista, N. Nuclear quantum effects on the thermodynamic, structural, and dynamical properties of water. Phys. Chem. Chem. Phys. 23, 6914-6928 (2021).

47. Ramírez, R., Neuerburg, N., Fernández-Serra, M.-V. \& Herrero, C. Quasi-harmonic approximation of thermodynamic properties of ice ih, ii, and iii. The J. chemical physics 137, 044502 (2012).

48. Pamuk, B., Allen, P. \& Fernández-Serra, M.-V. Insights into the structure of liquid water from nuclear quantum effects on the density and compressibility of ice polymorphs. The J. Phys. Chem. B 122, 5694-5706 (2018).

\section{Acknowledgements}

The authors are grateful to Vadim Brazhkin for the interest in this work and useful discussions. The authors acknowledge the Supercomputer Centre of JIHT RAS and the Supercomputer Centre of MIPT. This research was supported in part through computational resources of HPC facilities at NRU HSE. This work was prepared in part within the framework of the HSE University Basic Research Program.

\section{Author contributions statement}

V.S. conceived the study, A.G. made the necessary calculations, processed the data and wrote the manuscript. All authors analysed the results and reviewed and edited the manuscript.

\section{Competing interests}

The authors declare no competing interests.

\section{Additional information}

Supplementary information is available on the online version of the work.

Competing interests: The authors declare no competing interests.

Correspondence and requests for materials should be addressed to A.G.

Reprints and permission information is available online at www.nature.com/reprints..

Open Access This work is licensed under a Creative Commons Attribution 4.0 International License. The images or other third party material in this article are included in the article's Creative Commons license, unless indicated otherwise in the credit line; if the material is not included under the Creative Commons license, users will need to obtain permission from the license holder to reproduce the material. To view a copy of this license, visit http://creativecommons.org/licenses/by/4.0/ 


\section{Supplementary Files}

This is a list of supplementary files associated with this preprint. Click to download.

- HDALDAdecompression.mov

- HDALDAheating2880.mp4

- HDALDAheating77760.mp4

- LDAHDAcompression.mov

- Supplementary.pdf 